\title{
Oscillation for a generalized neutral Emden-Fowler equation with damping and distributed delay
}

\author{
Fengsheng Lei ${ }^{\mathrm{a}}$, Jiandong $\mathrm{Li}^{\mathrm{a}}$, Guirong Liu ${ }^{\mathrm{b}, *}$ \\ a Department of Mathematics, Luliang University, Lishi, Shanxi 033000 China \\ b School of Mathematical Sciences, Shanxi University, Taiyuan, Shanxi 030006 China
}

*Corresponding author, e-mail: lgr5791@sxu.edu.cn

Received 27 Sep 2018

Accepted 6 Aug 2019

ABSTRACT: In this study, we consider the generalized neutral Emden-Fowler equation with damping and distributed delay

$$
\left[r(t)\left|z^{\prime}(t)\right|^{\alpha-1} z^{\prime}(t)\right]^{\prime}+c(t)\left|z^{\prime}(t)\right|^{\alpha-1} z^{\prime}(t)+\int_{a}^{b} q(t, \xi)|x(g(t, \xi))|^{\beta-1} x(g(t, \xi)) \mathrm{d} \xi=0,
$$

where $z(t)=x(t)+p(t) x(\tau(t)), \alpha, \beta>0$. By using averaging technique and some analytical skills, we obtain the sufficient conditions to ensure the oscillation for the above equation. Our results improve and generalize some existing results. Finally, two examples are given to show the feasibility of our results.

KEYWORDS: oscillation, damping, distributed delay, Emden-Fowler equation

MSC2010: 34K11 34K40

\section{INTRODUCTION}

Since Emden-Fowler equations with generalized forms ${ }^{1-4}$ have many applications in various fields of nuclear physics, astrophysics and economics, there is constant interest in obtaining new sufficient conditions for oscillation of solutions of these equations $^{5-7}$. For more details of oscillation of delay differential equations ${ }^{8-10}$, neutral differential equations $^{11-14}$ and dynamic equations ${ }^{15-18}$, see the mentioned references.

In Ref. 19, Baculíková and Džurina studied the oscillation of the second-order neutral differential equation of the form

$$
\left[a(t)\left[z^{\prime}(t)\right]^{\alpha}\right]^{\prime}+q(t) x^{\beta}(\sigma(t))=0,
$$

where $z(t)=x(t)+p(t) x(\tau(t)), \alpha, \beta>0$.

In Ref. 20, Liu et al considered the generalized Emden-Fowler equation

$$
\left[r(t)\left|z^{\prime}(t)\right|^{\alpha-1} z^{\prime}(t)\right]^{\prime}+q(t)|x(\sigma(t))|^{\beta-1} x(\sigma(t))=0,
$$

where $t \geqslant t_{0}, z(t)=x(t)+p(t) x(\tau(t))$. By using averaging technique and Riccati transformation, they obtained some oscillation criteria for $\alpha \geqslant \beta>0$.

Subsequently, by using the similar method, Zeng et $\mathrm{al}^{21}$ obtained the oscillation for (2) with $\alpha \geqslant \beta>0$ or $0<\alpha \leqslant \beta$. Further, by using the generalized Riccati inequality, Wu et $\mathrm{al}^{22}$ studied the oscillation of (2) for $\alpha, \beta>0$.

In recent years, the oscillation of differential equations with damping or distributed delay has been studied by many authors. For example, Bohner et $\mathrm{al}^{23}$ studied the oscillation of the second-order damped nonlinear delay differential equations of Emden-Fowler type

$$
\begin{aligned}
& {\left[a(t) x^{\prime}(t)\right]^{\prime}+p(t) x^{\prime}(t)+} \\
& \quad q(t)|x(g(t))|^{\lambda} \operatorname{sgn} x(g(t))=0, t \in\left[t_{0}, \infty\right) .
\end{aligned}
$$

Zeng et $\mathrm{al}^{24}$ studied the oscillation of generalized neutral delay differential equations of EmdenFowler type with damping

$$
\begin{aligned}
& {\left[r(t)\left|z^{\prime}(t)\right|^{\alpha-1} z^{\prime}(t)\right]^{\prime}+c(t)\left|z^{\prime}(t)\right|^{\alpha-1} z^{\prime}(t)} \\
& \quad+q(t)|x(\sigma(t))|^{\beta-1} x(\sigma(t))=0, t \geqslant t_{0} .
\end{aligned}
$$

By using integral inequality technique and Riccati transformation, they obtained some oscillation criteria for the case $\alpha \geqslant \beta>0$ or $\beta \geqslant \alpha>0$.

Qin et $\mathrm{al}^{25}$ studied the second-order differential equation with distributed deviating arguments and 
a damping term

$$
\begin{aligned}
& r(x) \psi(y(x))[y(x)+c(x) y(d(x))]^{\prime} \\
&+\int_{\alpha}^{\beta} a(x, \theta) f(y(b(x, \theta))) \mathrm{d} \theta=0,
\end{aligned}
$$

and established some oscillation criteria of this equation.

In this study, motivated by the above work, by using averaging technique and some analytical skills, we obtain the sufficient conditions to ensure the oscillation for the following generalized EmdenFowler equation with damping and distributed delay

$$
\begin{aligned}
& {\left[r(t)\left|z^{\prime}(t)\right|^{\alpha-1} z^{\prime}(t)\right]^{\prime}+c(t)\left|z^{\prime}(t)\right|^{\alpha-1} z^{\prime}(t)} \\
& +\int_{a}^{b} q(t, \xi)|x(g(t, \xi))|^{\beta-1} x(g(t, \xi)) \mathrm{d} \xi=0,
\end{aligned}
$$

where $t \geqslant t_{0}, z(t)=x(t)+p(t) x(\tau(t))$.

In the sequel, we always make the following assumptions for (6).

$\left(\mathrm{H}_{1}\right) a, b, \alpha>0, \beta>0$ are all constants. In addition, $b>a, p \in C\left(\left[t_{0}, \infty\right),[0,1)\right), c \in$ $C\left(\left[t_{0}, \infty\right),[0, \infty)\right), r \in C^{1}\left(\left[t_{0}, \infty\right),(0, \infty)\right)$, $r^{\prime}(t) \geqslant 0$ for $t \in\left[t_{0}, \infty\right)$

$\left(\mathrm{H}_{2}\right) \tau \in C^{1}\left(\left[t_{0}, \infty\right), R\right), \quad q, g \in C\left(\left[t_{0}, \infty\right) \times\right.$ $[a, b],[0, \infty)), q(t, \xi) \not \equiv 0, \lim _{t \rightarrow \infty} g(t, \xi)=\infty$ uniformly holds on $\xi \in[a, b], 0 \leqslant \tau(t) \leqslant t$, $\lim _{t \rightarrow \infty} \tau(t)=\infty ; g(t, \xi) \leqslant t, \quad g_{t}(t, \xi)>0$, $g_{\xi}(t, \xi) \geqslant 0$ for $\xi \in[a, b], t \in\left[t_{0}, \infty\right)$;

$\left(\mathrm{H}_{3}\right) \lim _{t \rightarrow \infty} R(t)=\infty$, where

$$
R(t)=\int_{t_{0}}^{t}\left(r(\eta) \mathrm{e}^{\int_{t_{0}}^{\eta} \frac{c(s)}{r(s)} \mathrm{d} s}\right)^{-1 / \alpha} \mathrm{d} \eta
$$

$\left(\mathrm{H}_{4}\right)$ There exists $\rho \in C^{1}\left(\left[t_{0}, \infty\right),(0, \infty)\right)$ such that $\rho^{\prime}(t) \geqslant 0$ and for any $m \in(0,1]$,

$\int_{t_{0}}^{\infty}\left[\rho(t) Q(t)-\frac{r(t)\left[\rho^{\prime}(t)\right]^{\lambda+1} \mathrm{e}^{\int_{t_{0}}^{t} \frac{c(s)}{r(s)} \mathrm{d} s}}{(\lambda+1)^{\lambda+1}\left[m \rho(t) g_{t}(t, a)\right]^{\lambda}}\right] \mathrm{d} t=\infty$, where $\lambda=\min \{\alpha, \beta\}$,

$$
Q(t)=\mathrm{e}^{\int_{t_{0}}^{t} \frac{c(s)}{r(s)} \mathrm{d} s} \int_{a}^{b} q(t, \xi)[1-p(g(t, \xi))]^{\beta} \mathrm{d} \xi ;
$$

$\left(\mathrm{H}_{5}\right) \Pi(t)<\infty$ for $t \in\left[t_{0}, \infty\right)$, where

$$
\Pi(t)=\int_{t}^{\infty}\left[r(\eta) \mathrm{e}^{\int_{t_{0}}^{\eta} \frac{c(s)}{r(s)} \mathrm{d} s}\right]^{-1 / \alpha} \mathrm{d} \eta .
$$

\section{MAIN RESULTS}

It is clear that (6) is equivalent to the following equation

$$
\begin{aligned}
& {\left[\mathrm{e}^{\int_{t_{0}}^{t} \frac{c(s)}{r(s)} \mathrm{d} s} r(t)\left|z^{\prime}(t)\right|^{\alpha-1} z^{\prime}(t)\right]^{\prime}+\mathrm{e}^{\int_{t_{0}}^{t} \frac{c(s)}{r(s)} \mathrm{d} s}} \\
& \times \int_{a}^{b} q(t, \xi)|x(g(t, \xi))|^{\beta-1} x(g(t, \xi)) \mathrm{d} \xi=0,
\end{aligned}
$$

where $t \geqslant t_{0}$. Therefore, the oscillation of (6) is equivalent to that of (7). In order to prove the main results, we need the following lemmas.

Lemma 1 Let $\theta, A, B$ be constants, $\theta>0, A \geqslant 0$, $B>0$. Then, for any $u>0$,

$$
A u-B u^{(\theta+1) / \theta} \leqslant \frac{\theta^{\theta}}{(\theta+1)^{\theta+1}} \frac{A^{\theta+1}}{B^{\theta}} .
$$

The proof of Lemma 1 is easy. So we omit it here.

Lemma 2 Suppose that $\left(\mathrm{H}_{1}\right),\left(\mathrm{H}_{2}\right)$, and $\left(\mathrm{H}_{5}\right)$ hold. Let $x(t)$ be an eventually positive solution for (7). In addition, $z^{\prime}(t)$ is eventually negative. Then there exist $T>t_{0}$ and $L>0$ such that

$$
0<w_{1}(t) \Pi^{\mu}(t) \leqslant L, \quad t \in[T, \infty),
$$

where $\mu=\max \{\alpha, \beta\}$,

$$
w_{1}(t)=\mathrm{e}^{\int_{t_{0}}^{t} \frac{c(s)}{r(s)} \mathrm{d} s} r(t)\left(-z^{\prime}(t)\right)^{\alpha} / z^{\beta}(t) .
$$

Proof: From $\left(\mathrm{H}_{1}\right), z(t)$ is eventually positive. Further, by using (7) and $\left(\mathrm{H}_{2}\right)$, there exists $T>t_{0}$ such that

$$
\left[\mathrm{e}^{\int_{t_{0}}^{t} \frac{c(s)}{r(s)} \mathrm{d} s} r(t)\left(-z^{\prime}(t)\right)^{\alpha}\right]^{\prime} \geqslant 0, \quad t \in[T, \infty) .
$$

Hence, for any $v>t>T$, we have

$$
-z^{\prime}(v)\left[\mathrm{e}^{\int_{t_{0}}^{v} \frac{c(s)}{r(s)} \mathrm{d} s} r(v)\right]^{1 / \alpha} \geqslant-z^{\prime}(t)\left[\mathrm{e}^{\int_{t_{0}}^{t} \frac{c(s)}{r(s)} \mathrm{d} s} r(t)\right]^{1 / \alpha},
$$

which yields

$$
-z^{\prime}(v) \geqslant-z^{\prime}(t)\left[\mathrm{e}^{\int_{t_{0}}^{v} \frac{c(s)}{r(s)} \mathrm{d} s} r(v)\right]^{-1 / \alpha}\left[\mathrm{e}^{\int_{t_{0}}^{t} \frac{c(s)}{r(s)} \mathrm{d} s} r(t)\right]^{1 / \alpha} .
$$

Further, for any $u>t>T$, we have

$$
\begin{aligned}
z(t) \geqslant z(t) & -z(u) \geqslant\left[\mathrm{e}^{\int_{t_{0}}^{t} \frac{c(s)}{r(s)} \mathrm{d} s} r(t)\right]^{1 / \alpha} \\
& \times\left(-z^{\prime}(t)\right) \int_{t}^{u}\left[\mathrm{e}^{\int_{t_{0}}^{v} \frac{c(s)}{r(s)} \mathrm{d} s} r(v)\right]^{-1 / \alpha} \mathrm{d} v .
\end{aligned}
$$


Letting $u \rightarrow \infty$ in (9) yields

$z(t) \geqslant\left[\mathrm{e}^{\int_{t_{0}}^{t} \frac{c(s)}{r(s)} \mathrm{d} s} r(t)\right]^{1 / \alpha}\left(-z^{\prime}(t)\right) \Pi(t), \quad t \in[T, \infty)$.

Further,

$$
\begin{aligned}
& z^{\alpha}(t) \geqslant r(t) \mathrm{e}^{\int_{t_{0}}^{t} \frac{c(s)}{r(s)} \mathrm{d} s}\left(-z^{\prime}(t)\right)^{\alpha} \Pi^{\alpha}(t), \\
& z^{\beta}(t) \geqslant\left[\mathrm{e}^{\int_{t_{0}}^{t} \frac{c(s)}{r(s)} \mathrm{d} s} r(t)\right]^{\beta / \alpha}\left(-z^{\prime}(t)\right)^{\beta} \Pi^{\beta}(t) .
\end{aligned}
$$

If $\alpha \geqslant \beta$, then $\mu=\alpha$ and

$$
\begin{aligned}
& w_{1}(t) \Pi^{\mu}(t)=w_{1}(t) \Pi^{\alpha}(t) \\
& \leqslant \frac{\mathrm{e}^{\int_{t_{0}}^{t} \frac{c(s)}{r(s)} \mathrm{d} s} r(t)\left(-z^{\prime}(t)\right)^{\alpha}}{z^{\beta}(t)} \frac{z^{\alpha}(t)}{\mathrm{e}^{\int_{t_{0}}^{t} \frac{c(s)}{r(s)} \mathrm{d} s} r(t)\left(-z^{\prime}(t)\right)^{\alpha}} \\
& =z^{\alpha-\beta}(t) .
\end{aligned}
$$

Note that $z(t)>0$ and $z^{\prime}(t)<0$. There exists a constant $L_{1}>0$ such that

$$
0<w_{1}(t) \Pi^{\mu}(t) \leqslant z^{\alpha-\beta}(t) \leqslant L_{1}, \quad t \in[T, \infty) .
$$

If $\alpha<\beta$, then $\mu=\beta$. Therefore, for $t \in[T, \infty)$,

$$
\begin{aligned}
& w_{1}(t) \Pi^{\mu}(t)=w_{1}(t) \Pi^{\beta}(t) \\
& \leqslant \frac{\mathrm{e}^{\int_{t_{0}}^{t} \frac{c(s)}{r(s)} \mathrm{d} s} r(t)\left(-z^{\prime}(t)\right)^{\alpha}}{z^{\beta}(t)} \frac{z^{\beta}(t)}{\left[\mathrm{e}^{\int_{t_{0}}^{t} \frac{c(s)}{r(s)} \mathrm{d} s} r(t)\right]^{\frac{\beta}{\alpha}}\left(-z^{\prime}(t)\right)^{\beta}} \\
& =\left[\mathrm{e}^{\int_{t_{0}}^{t} \frac{c(s)}{r(s)} \mathrm{d} s} r(t)\left(-z^{\prime}(t)\right)^{\alpha}\right]^{\frac{\alpha-\beta}{\alpha}} .
\end{aligned}
$$

From (8), there exists a constant $L_{2}>0$ such that

$$
0<w_{1}(t) \Pi^{\mu}(t) \leqslant L_{2}, \quad t \in[T, \infty) .
$$

Denote $L=\max \left\{L_{1}, L_{2}\right\}$. Hence, for any $t \in[T, \infty)$,

$$
0<w_{1}(t) \Pi^{\mu}(t) \leqslant L .
$$

This completes the proof of Lemma 2 .

Theorem 1 Assume that $\left(\mathrm{H}_{1}\right)-\left(\mathrm{H}_{4}\right)$ hold. Then (6) is oscillatory.

Proof: Suppose that (7) has a nonoscillatory solution $x(t)$. Without loss of generality, we assume that $x(t)$ is an eventually positive solution for (7). Hence, there exists $T_{0}>t_{0}$ such that $x(t)>0$ for $t \geqslant T_{0}$. If $x(t)$ is eventually negative, the proof is similar.

From $\left(\mathrm{H}_{2}\right)$ and (7), there exists $T_{1} \geqslant T_{0}$ such that $\tau(t) \geqslant T_{0}$ and $g(t, \xi) \geqslant T_{0}$ for any $t \geqslant T_{1}$ and $\xi \in[a, b]$. This, together with $\left(\mathrm{H}_{1}\right)$ and (7), implies that for $t \geqslant T_{1}$,

$$
\begin{gathered}
x(g(t, \xi))>0, \quad x(\tau(t))>0, \quad z(t) \geqslant x(t)>0, \\
{\left[\mathrm{e}^{\int_{t_{0}}^{t} \frac{c(s)}{r(s)} \mathrm{d} s} r(t)\left|z^{\prime}(t)\right|^{\alpha-1} z^{\prime}(t)\right]^{\prime} \leqslant 0 .}
\end{gathered}
$$

Further, from (10), it is easy to see that $z^{\prime}(t)$ is eventually nonnegative or eventually negative. Suppose that $z^{\prime}(t)$ is eventually negative. That is, there exists $T_{2} \geqslant T_{1}$ such that $z^{\prime}(t)<0$ for any $t \geqslant T_{2}$. It follows from (10) that

$$
\begin{gathered}
{\left[-\mathrm{e}^{\int_{t_{0}}^{t} \frac{c(s)}{r(s)} \mathrm{d} s} r(t)\left(-z^{\prime}(t)\right)^{\alpha}\right]^{\prime} \leqslant 0, \quad t \geqslant T_{2} .} \\
\text { Denote } K=r\left(T_{2}\right)\left(-z^{\prime}\left(T_{2}\right)\right)^{\alpha} \mathrm{e}^{\int_{t_{0}}^{T_{2}} \frac{c(s)}{r(s)} \mathrm{d} s}>0 . \text { Hence, } \\
-\mathrm{e}^{\int_{t_{0}}^{t} \frac{c(s)}{r(s)} \mathrm{d} s} r(t)\left(-z^{\prime}(t)\right)^{\alpha} \leqslant-K, \quad t \geqslant T_{2} .
\end{gathered}
$$

This yields

$$
-z^{\prime}(t) \geqslant K^{1 / \alpha}\left[r(t) \mathrm{e}^{\int_{t_{0}}^{t} \frac{c(s)}{r(s)} \mathrm{d} s}\right]^{-1 / \alpha}, \quad t \geqslant T_{2} .
$$

Integrating this inequality from $T_{2}$ to $t$, we obtain

$$
z(t) \leqslant z\left(T_{2}\right)-K^{1 / \alpha}\left[R(t)-R\left(T_{2}\right)\right], \quad t \geqslant T_{2} .
$$

It follows from $\left(\mathrm{H}_{3}\right)$ that $\lim _{t \rightarrow \infty} z(t)=-\infty$. This contradicts (10). Therefore $z^{\prime}(t)$ is eventually nonnegative. That is, there exists $T_{3} \geqslant T_{2}$ such that $z^{\prime}(t) \geqslant 0$ and $z^{\prime}(g(t, \xi)) \geqslant 0$ for any $t \geqslant T_{3}$. Further, (7) yields

$$
\begin{aligned}
& {\left[\mathrm{e}^{\int_{t_{0}}^{t} \frac{c(s)}{r(s)} \mathrm{d} s} r(t)\left(z^{\prime}(t)\right)^{\alpha}\right]^{\prime}+\mathrm{e}^{\int_{t_{0}}^{t} \frac{c(s)}{r(s)} \mathrm{d} s}} \\
& \quad \times \int_{a}^{b} q(t, \xi) x^{\beta}(g(t, \xi)) \mathrm{d} \xi=0, \quad t \geqslant T_{3} .
\end{aligned}
$$

From (10), for $t \geqslant T_{3}$, we have

$$
\begin{aligned}
& {\left[\mathrm{e}^{\int_{t_{0}}^{t} \frac{c(s)}{r(s)} \mathrm{d} s} r(t)\left(z^{\prime}(t)\right)^{\alpha}\right]^{\prime}} \\
& =\mathrm{e}^{\int_{t_{0}}^{t} \frac{c(s)}{r(s)} \mathrm{d} s}\left[c(t)\left(z^{\prime}(t)\right)^{\alpha}+r^{\prime}(t)\left(z^{\prime}(t)\right)^{\alpha}\right. \\
& \left.\quad+r(t) \alpha\left(z^{\prime}(t)\right)^{\alpha-1} z^{\prime \prime}(t)\right] \leqslant 0,
\end{aligned}
$$

which, together with $\left(\mathrm{H}_{1}\right)$ and (10), yields

$$
z^{\prime \prime}(t) \leqslant 0, \quad t \geqslant T_{3} .
$$

Hence, there exists $T_{4} \geqslant T_{3}$ such that

$$
z^{\prime}(t)>0, t \geqslant T_{4} ; \quad \text { or } \quad z^{\prime}(t) \equiv 0, t \geqslant T_{4} .
$$


In fact, if $z^{\prime}(t) \equiv 0$ for $t \geqslant T_{4}$, then it follows from (7) that $q(t, \xi) \equiv 0$ for $t \geqslant T_{4}$ and $\xi \in[a, b]$. This contradicts $\left(\mathrm{H}_{2}\right)$. Hence

$$
z^{\prime}(t)>0, \quad t \geqslant T_{4}
$$

It follows from $z(t) \geqslant x(t)$ that

$$
\begin{aligned}
x(t)=z(t)-p(t) x(\tau(t)) & \geqslant z(t)-p(t) z(\tau(t)) \\
& \geqslant[1-p(t)] z(t), \quad t \geqslant T_{4} .
\end{aligned}
$$

Therefore

$$
x^{\beta}(t) \geqslant[1-p(t)]^{\beta} z^{\beta}(t), \quad t \geqslant T_{4} .
$$

From $\left(\mathrm{H}_{1}\right)$ and $\left(\mathrm{H}_{2}\right)$, there exists $T_{5} \geqslant T_{4}$ such that

$$
x^{\beta}(g(t, \xi)) \geqslant[1-p(g(t, \xi))]^{\beta} z^{\beta}(g(t, \xi)),
$$$$
t \geqslant T_{5}, \xi \in[a, b] \text {. }
$$

From $\left(\mathrm{H}_{2}\right)$, for $t \geqslant T_{5}$,

$$
\begin{aligned}
& \int_{a}^{b} q(t, \xi) x^{\beta}(g(t, \xi)) \mathrm{d} \xi \\
& \geqslant \int_{a}^{b} q(t, \xi)[1-p(g(t, \xi))]^{\beta} z^{\beta}(g(t, \xi)) \mathrm{d} \xi \\
& \geqslant z^{\beta}(g(t, a)) \int_{a}^{b} q(t, \xi)[1-p(g(t, \xi))]^{\beta} \mathrm{d} \xi .
\end{aligned}
$$

It follows from (11) and (13) that for $t \geqslant T_{5}$,

$$
\begin{aligned}
& {\left[\mathrm{e}^{\int_{t_{0}}^{t} \frac{c(s)}{r(s)} \mathrm{d} s} r(t)\left(z^{\prime}(t)\right)^{\alpha}\right]^{\prime}+\mathrm{e}^{\int_{t_{0}}^{t} \frac{c(s)}{r(s)} \mathrm{d} s}} \\
& \quad \times z^{\beta}(g(t, a)) \int_{a}^{b} q(t, \xi)[1-p(g(t, \xi))]^{\beta} \mathrm{d} \xi \leqslant 0 .
\end{aligned}
$$

Hence,

$$
\frac{\left[\mathrm{e}^{\int_{t_{0}}^{t} \frac{c(s)}{r(s)} \mathrm{d} s} r(t)\left(z^{\prime}(t)\right)^{\alpha}\right]^{\prime}}{z^{\beta}(g(t, a))} \leqslant-Q(t), \quad t \geqslant T_{5} .
$$

Denote

$$
w(t)=\rho(t) \frac{\mathrm{e}^{\int_{t_{0}}^{t} \frac{c(s)}{r(s)} d s} r(t)\left(z^{\prime}(t)\right)^{\alpha}}{z^{\beta}(g(t, a))} \geqslant 0, t \geqslant T_{5} .
$$

Further,

$$
\begin{gathered}
w^{\frac{\alpha+1}{\alpha}}(t) \\
=\frac{\mathrm{e}^{\int_{t_{0}}^{t} \frac{c(s)}{r(s)} \mathrm{d} s} \rho(t) r(t)\left[\mathrm{e}^{\int_{t_{0}}^{t} \frac{c(s)}{r(s)} \mathrm{d} s} \rho(t) r(t)\right]^{\frac{1}{\alpha}}\left(z^{\prime}(t)\right)^{\alpha+1}}{z^{\frac{\beta(\alpha+1)}{\alpha}}(g(t, a))}, \\
t \geqslant T_{5}, \quad \text { (16) }
\end{gathered}
$$

and

$$
\begin{aligned}
& w^{\frac{\beta+1}{\beta}}(t) \\
& =\frac{\mathrm{e}^{\int_{t_{0}}^{t} \frac{c(s)}{r(s)} \mathrm{d} s} \rho(t) r(t)\left[\mathrm{e}^{\int_{t_{0}}^{t} \frac{c(s)}{r(s)} \mathrm{d} s} \rho(t) r(t)\right]^{\frac{1}{\beta}}\left(z^{\prime}(t)\right)^{\frac{\alpha(\beta+1)}{\beta}}}{z^{\beta+1}(g(t, a))}, \\
& t \geqslant T_{5} .
\end{aligned}
$$

In addition, for $t \geqslant T_{5}$,

$$
\begin{gathered}
w^{\prime}(t)=\frac{\rho^{\prime}(t)}{\rho(t)} w(t)+\rho(t) \frac{\left[\mathrm{e}^{\int_{t_{0}}^{t} \frac{c(s)}{r(s)} \mathrm{d} s} r(t)\left(z^{\prime}(t)\right)^{\alpha}\right]^{\prime}}{z^{\beta}(g(t, a))} \\
-\rho(t) \frac{\beta \mathrm{e}^{\int_{t_{0}}^{t} \frac{c(s)}{r(s)} \mathrm{d} s} r(t)\left[z^{\prime}(t)\right]^{\alpha} z^{\prime}(g(t, a)) g_{t}(t, a)}{z^{\beta+1}(g(t, a))}
\end{gathered}
$$

If $\alpha=\beta$, then it follows from $\left(\mathrm{H}_{2}\right),(12),(14),(16)$, and (18) that for $t \geqslant T_{5}$,

$$
\begin{aligned}
& w^{\prime}(t)= \frac{\rho^{\prime}(t)}{\rho(t)} w(t)+\rho(t) \frac{\left[\mathrm{e}^{\int_{t_{0}}^{t} \frac{c(s)}{r(s)} \mathrm{d} s} r(t)\left(z^{\prime}(t)\right)^{\alpha}\right]^{\prime}}{z^{\beta}(g(t, a))} \\
&-\rho(t) \frac{\alpha \mathrm{e}^{\int_{t_{0}}^{t} \frac{c(s)}{r(s)} \mathrm{d} s} r(t)\left[z^{\prime}(t)\right]^{\alpha} z^{\prime}(g(t, a)) g_{t}(t, a)}{z^{\alpha+1}(g(t, a))} \\
& \leqslant \frac{\rho^{\prime}(t)}{\rho(t)} w(t)-\rho(t) Q(t) \\
&-w^{\frac{\alpha+1}{\alpha}}(t) \frac{\alpha z^{\prime}(g(t, a)) g_{t}(t, a)}{\left[\mathrm{e}^{\int_{t_{0}}^{t} \frac{c(s)}{r(s)} \mathrm{d} s} \rho(t) r(t)\right]^{\frac{1}{\alpha}} z^{\prime}(t)} \\
& \leqslant \frac{\rho^{\prime}(t)}{\rho(t)} w(t)-\rho(t) Q(t) \\
&-w^{\frac{\alpha+1}{\alpha}}(t) \frac{\alpha g_{t}(t, a)}{\left[\mathrm{e}^{\int_{t_{0}}^{t} \frac{c(s)}{r(s)} \mathrm{d} s} \rho(t) r(t)\right]^{\frac{1}{\alpha}}} .
\end{aligned}
$$

If $\alpha<\beta$, then it follows from (16) and (18) that for $t \geqslant T_{5}$,

$$
\begin{gathered}
w^{\prime}(t)=\frac{\rho^{\prime}(t)}{\rho(t)} w(t)+\rho(t) \frac{\left[\mathrm{e}^{\int_{t_{0}}^{t} \frac{c(s)}{r(s)} \mathrm{d} s} r(t)\left(z^{\prime}(t)\right)^{\alpha}\right]^{\prime}}{z^{\beta}(g(t, a))} \\
\left.-w^{\frac{\alpha+1}{\alpha}}(t) \frac{\beta z^{\prime}(g(t, a)) g_{t}(t, a)[z(g(t, a))]^{\frac{\beta-\alpha}{\alpha}}}{\left[\mathrm{e}^{t} \frac{c(s)}{t_{0}(s)} \mathrm{d} s\right.} \rho(t) r(t)\right]^{\frac{1}{\alpha}} z^{\prime}(t)
\end{gathered}
$$

Note that $z^{\prime}(t)>0$ for $t \geqslant T_{4}$. From $\left(\mathrm{H}_{2}\right)$, we have

$[z(g(t, a))]^{\frac{\beta-\alpha}{\alpha}} \geqslant\left[z\left(g\left(T_{5}, a\right)\right)\right]^{\frac{\beta-\alpha}{\alpha}}=m_{1}, t \geqslant T_{5} .(21)$ 
Obviously, $m_{1}>0$. From (12), (14), (20), and (21), Integrating (26) from $T_{5}$ to $t$, we get

it follows that for $t \geqslant T_{5}$,

$$
\begin{aligned}
w^{\prime}(t) \leqslant & \frac{\rho^{\prime}(t)}{\rho(t)} w(t)-\rho(t) Q(t) \\
& -w^{\frac{\alpha+1}{\alpha}}(t) \frac{\beta z^{\prime}(g(t, a)) g_{t}(t, a)[z(g(t, a))]^{\frac{\beta-\alpha}{\alpha}}}{\left[\mathrm{e}^{\int_{t_{0}}^{t} \frac{c(s)}{r(s)} \mathrm{d} s} \rho(t) r(t)\right]^{\frac{1}{\alpha}} z^{\prime}(t)} \\
\leqslant & \frac{\rho^{\prime}(t)}{\rho(t)} w(t)-\rho(t) Q(t) \\
& -w^{\frac{\alpha+1}{\alpha}}(t) m_{1} \frac{\alpha g_{t}(t, a)}{\left[\mathrm{e}^{\int_{t_{0}}^{t} \frac{c(s)}{r(s)} \mathrm{d} s} \rho(t) r(t)\right]^{\frac{1}{\alpha}}} \cdot \quad(22)
\end{aligned}
$$

If $\alpha>\beta$, it follows from (17) and (18), for $t \geqslant T_{5}$,

$$
\begin{aligned}
& w^{\prime}(t)=\frac{\rho^{\prime}(t)}{\rho(t)} w(t)+\rho(t) \frac{\left[\mathrm{e}^{\int_{t_{0}}^{t} \frac{c(s)}{r(s)} \mathrm{d} s} r(t)\left(z^{\prime}(t)\right)^{\alpha}\right]^{\prime}}{z^{\beta}(g(t, a))} \\
&-w^{\frac{\beta+1}{\beta}}(t) \frac{\beta z^{\prime}(g(t, a)) g_{t}(t, a)\left[z^{\prime}(t)\right]^{\frac{\beta-\alpha}{\beta}}}{\left[\mathrm{e}^{\int_{t_{0}}^{t} \frac{c(s)}{r(s)} \mathrm{d} s} \rho(t) r(t)\right]^{\frac{1}{\beta}} z^{\prime}(t)}
\end{aligned}
$$

From (12), we have

$$
\left[z^{\prime}(t)\right]^{\frac{\beta-\alpha}{\beta}} \geqslant\left[z^{\prime}\left(T_{5}\right)\right]^{\frac{\beta-\alpha}{\beta}}=m_{2}, \quad t \geqslant T_{5} .
$$

Further, $m_{2}>0$. From (12), (14), (23), and (24), for $t \geqslant T_{5}$, we have

$$
\begin{aligned}
w^{\prime}(t) \leqslant & \frac{\rho^{\prime}(t)}{\rho(t)} w(t)-\rho(t) Q(t) \\
& -w^{\frac{\beta+1}{\beta}}(t) \frac{\beta g_{t}(t, a)\left[z^{\prime}(t)\right]^{\frac{\beta-\alpha}{\beta}}}{\left[\mathrm{e}^{\int_{t_{0}}^{t} \frac{c(s)}{r(s)} \mathrm{d} s} \rho(t) r(t)\right]^{\frac{1}{\beta}}} \\
\leqslant & \frac{\rho^{\prime}(t)}{\rho(t)} w(t)-\rho(t) Q(t) \\
& -w^{\frac{\beta+1}{\beta}}(t) m_{2} \frac{\beta g_{t}(t, a)}{\left[\mathrm{e}^{\int_{t_{0}}^{t} \frac{c(s)}{r(s)} \mathrm{d} s} \rho(t) r(t)\right]^{\frac{1}{\beta}}} .
\end{aligned}
$$

From (19), (22), (25), and Lemma 1, there exists $m \in(0,1]$ such that

$$
\begin{aligned}
& w^{\prime}(t) \leqslant-\rho(t) Q(t)+\frac{\rho^{\prime}(t)}{\rho(t)} w(t) \\
& -w^{\frac{\lambda+1}{\lambda}}(t) \frac{\lambda m g_{t}(t, a)}{\left[\mathrm{e}^{\int_{t_{0}}^{t} \frac{c(s)}{r(s)} \mathrm{d} s} \rho(t) r(t)\right]^{\frac{1}{\lambda}}} \\
& \leqslant-\rho(t) Q(t)+\frac{r(t)\left(\rho^{\prime}(t)\right)^{\lambda+1} \mathrm{e}^{\int_{t_{0}}^{t} \frac{c(s)}{r(s)} \mathrm{d} s}}{(\lambda+1)^{\lambda+1}\left(m g_{t}(t, a) \rho(t)\right)^{\lambda}} .
\end{aligned}
$$

$w(t) \leqslant w\left(T_{5}\right)-$

$\int_{T_{5}}^{t}\left[\rho(s) Q(s)-\frac{r(s)\left[\rho^{\prime}(s)\right]^{\lambda+1} \mathrm{e}^{\int_{t_{0}}^{s} \frac{c(k)}{r(k)} \mathrm{d} k}}{(\lambda+1)^{\lambda+1}\left[m g_{s}(s, a) \rho(s)\right]^{\lambda}}\right] \mathrm{d} s$.

Letting $t \rightarrow \infty$ in (27), it follows from $\left(\mathrm{H}_{4}\right)$ that $\lim _{t \rightarrow \infty} w(t)=-\infty$. This contradicts (15). Therefore, (7) is oscillatory. This completes the proof. Choosing $\rho(t)=1$ in Theorem 1 , we get the results.

Corollary 1 Assume that $\left(\mathrm{H}_{1}\right)-\left(\mathrm{H}_{3}\right)$ hold. If

$\int_{t_{0}}^{\infty}\left[\mathrm{e}^{\int_{t_{0}}^{t} \frac{c(s)}{r(s)} \mathrm{d} s} \int_{a}^{b} q(t, \xi)[1-p(g(t, \xi))]^{\beta} \mathrm{d} \xi\right] \mathrm{d} t=\infty$, then (6) is oscillatory.

Theorem 2 Assume that $\left(\mathrm{H}_{1}\right),\left(\mathrm{H}_{2}\right),\left(\mathrm{H}_{4}\right)$, and $\left(\mathrm{H}_{5}\right)$ hold. If

(i) $p^{\prime}(t) \geqslant 0, \tau^{\prime}(t) \geqslant 0, g(t, \xi) \leqslant \tau(t)$, for $t \in$ $\left[t_{0}, \infty\right), \xi \in[a, b]$

(ii) for any $M \in(0,1]$,

$$
\begin{aligned}
& \int_{t_{0}}^{\infty}\left[\Pi^{\mu}(t) Q_{1}(t)-\right. \\
& \frac{\mu^{2 \mu+1}}{\left.(\beta M)^{\mu} \Pi(t)(\mu+1)^{\mu+1}\left[\mathrm{e}^{\int_{t_{0}}^{t} \frac{c(s)}{r(s)} \mathrm{d} s} r(t)\right]^{\frac{1}{\alpha}}\right] \mathrm{d} t=\infty,} \\
& \text { where } \mu=\max \{\alpha, \beta\} \text { and } \\
& Q_{1}(t)=[1-p(t)]^{\beta} \mathrm{e}^{\int_{t_{0}}^{t} \frac{c(s)}{r(s)} \mathrm{d} s} \int_{a}^{b} q(t, \xi) \mathrm{d} \xi,
\end{aligned}
$$

then (6) is oscillatory.

Proof: Suppose that (7) has a nonoscillatory solution $x(t)$. Without loss of generality, we assume that $x(t)$ is an eventually positive solution for (7). Hence, there exists $T_{0}>t_{0}$ such that $x(t)>0$ for $t \geqslant T_{0}$. If $x(t)$ is eventually negative, the proof is similar.

From $\left(\mathrm{H}_{2}\right)$ and (7), there exists $T_{1} \geqslant T_{0}$ such that $\tau(t) \geqslant T_{0}$ and $g(t, \xi) \geqslant T_{0}$ for any $t \geqslant T_{1}$ and $\xi \in[a, b]$. This, together with $\left(\mathrm{H}_{1}\right)$ and (7), implies that for $t \geqslant T_{1}$

$$
\begin{gathered}
x(g(t, \xi))>0, \quad x(\tau(t))>0, \quad z(t) \geqslant x(t)>0, \\
{\left[\mathrm{e}^{\int_{t_{0}}^{t} \frac{c(s)}{r(s)} \mathrm{d} s} r(t)\left|z^{\prime}(t)\right|^{\alpha-1} z^{\prime}(t)\right]^{\prime} \leqslant 0 .}
\end{gathered}
$$


From (28), it is easy to see that $z^{\prime}(t)$ is eventually nonnegative or eventually negative.

Case 1 . Assume that $z^{\prime}(t)$ is eventually nonnegative. By using the same method as in the proof of Theorem 1, we can get a contradiction.

Case 2 . Assume that $z^{\prime}(t)$ is eventually negative. Hence there exists $T_{2} \geqslant T_{1}$ satisfying $z^{\prime}(t)<0$ for any $t \geqslant T_{2}$. It follows from (7) and (28) that for $t \geqslant T_{2}$,

$$
\begin{aligned}
& {\left[\mathrm{e}^{\int_{t_{0}}^{t} \frac{c(s)}{r(s)} \mathrm{d} s} r(t)\left(-z^{\prime}(t)\right)^{\alpha}\right]^{\prime}} \\
& \quad-\mathrm{e}^{\int_{t_{0}}^{t} \frac{c(s)}{r(s)} \mathrm{d} s} \int_{a}^{b} q(t, \xi) x^{\beta}(g(t, \xi)) \mathrm{d} \xi=0 .
\end{aligned}
$$

In addition,

$$
z^{\prime}(t)=x^{\prime}(t)+p^{\prime}(t) x(\tau(t))+p(t) x^{\prime}(\tau(t)) \tau^{\prime}(t),
$$

which together with $\left(\mathrm{H}_{1}\right)$ and condition $(i)$, yields

$$
x^{\prime}(t) \leqslant 0, \quad t \geqslant T_{2} .
$$

From (31) and $\left(\mathrm{H}_{2}\right)$, we deduce

$$
\begin{aligned}
z(t) & =x(t)+p(t) x(\tau(t))<x(\tau(t))+p(t) x(\tau(t)) \\
& =[1+p(t)] x(\tau(t)), \quad t \geqslant T_{2} .
\end{aligned}
$$

It follows from (31), (32) and ( $i$ ) that for any $\xi \in$ $[a, b]$,

$$
\begin{aligned}
x(g(t, \xi)) & \geqslant x(\tau(t))>\frac{z(t)}{1+p(t)}=\frac{z(t)(1-p(t))}{1-p^{2}(t)} \\
& \geqslant(1-p(t)) z(t), \quad t \geqslant T_{2} .
\end{aligned}
$$

From $\left(\mathrm{H}_{2}\right)$, for $t \geqslant T_{2}$, we have

$$
\begin{aligned}
\int_{a}^{b} q(t, \xi) x^{\beta}(g(t, \xi)) \mathrm{d} \xi \\
\geqslant \int_{a}^{b} q(t, \xi)[1-p(t)]^{\beta} z^{\beta}(t) \mathrm{d} \xi \\
\geqslant[1-p(t)]^{\beta} z^{\beta}(t) \int_{a}^{b} q(t, \xi) \mathrm{d} \xi .
\end{aligned}
$$

From (29) and (33), we get for $t \geqslant T_{2}$,

$$
\left[\mathrm{e}^{\int_{t_{0}}^{t} \frac{c(s)}{r(s)} d s} r(t)\left(-z^{\prime}(t)\right)^{\alpha}\right]^{\prime}-Q_{1}(t) z^{\beta}(t) \geqslant 0 .
$$

Clearly, for $t \geqslant T_{2}$,

$$
w_{1}(t):=\frac{\mathrm{e}^{\int_{t_{0}}^{t} \frac{c(s)}{r(s)} d s} r(t)\left(-z^{\prime}(t)\right)^{\alpha}}{z^{\beta}(t)}>0 .
$$

Further, for $t \geqslant T_{2}$,

$$
\begin{aligned}
w_{1}^{\frac{\alpha+1}{\alpha}}(t) & =\frac{\left[\mathrm{e}^{\int_{t_{0}}^{t} \frac{c(s)}{r(s)} \mathrm{d} s} r(t)\right]^{\frac{\alpha+1}{\alpha}}\left[-z^{\prime}(t)\right]^{\alpha+1}}{z^{\frac{\beta(\alpha+1)}{\alpha}}(t)}, \\
w_{1}^{\frac{\beta+1}{\beta}}(t) & =\frac{\left[\mathrm{e}^{\int_{t_{0}}^{t} \frac{c(s)}{r(s)} \mathrm{d} s} r(t)\right]^{\frac{\beta+1}{\beta}}\left[-z^{\prime}(t)\right]^{\frac{\alpha(\beta+1)}{\beta}}}{z^{\beta+1}(t)} .
\end{aligned}
$$

It follows from (34), (35) that for any $t \geqslant T_{2}$,

$$
\begin{aligned}
w_{1}^{\prime}(t)= & \frac{\left[\mathrm{e}^{\int_{t_{0}}^{t} \frac{c(s)}{r(s)} \mathrm{d} s} r(t)\left(-z^{\prime}(t)\right)^{\alpha}\right]^{\prime}}{z^{\beta}(t)} \\
& -\frac{\beta \mathrm{e}^{\int_{t_{0}}^{t} \frac{c(s)}{r(s)} \mathrm{d} s} z^{\beta-1}(t) z^{\prime}(t) r(t)\left(-z^{\prime}(t)\right)^{\alpha}}{z^{2 \beta}(t)} \\
\geqslant & Q_{1}(t)+\frac{\beta r(t)\left(-z^{\prime}(t)\right)^{\alpha+1} \mathrm{e}^{\int_{t_{0}}^{t} \frac{c(s)}{r(s)} \mathrm{d} s}}{z^{\beta+1}(t)} .
\end{aligned}
$$

If $\alpha \geqslant \beta$, then from (36) and (38) for $t \geqslant T_{2}$,

$$
w_{1}^{\prime}(t) \geqslant Q_{1}(t)+w_{1}^{\frac{\alpha+1}{\alpha}}(t) \frac{\beta[z(t)]^{\frac{\beta-\alpha}{\alpha}}}{\left[\mathrm{e}^{\int_{t_{0}}^{t} \frac{c(s)}{r(s)} \mathrm{d} s} r(t)\right]^{\frac{1}{\alpha}}} .
$$

Note that $z^{\prime}(t)<0$ for $t \geqslant T_{2}$. Therefore

$$
[z(t)]^{\frac{\beta-\alpha}{\alpha}} \geqslant\left[z\left(T_{2}\right)\right]^{\frac{\beta-\alpha}{\alpha}}=m_{3}, \quad t \geqslant T_{2} .
$$

Further, $m_{3}>0$. From (39) and (40) for $t \geqslant T_{2}$,

$$
w_{1}^{\prime}(t) \geqslant Q_{1}(t)+w_{1}^{\frac{\alpha+1}{\alpha}}(t) \frac{\beta m_{3}}{\left[\mathrm{e}^{\int_{t_{0}}^{t} \frac{c(s)}{r(s)} d s} r(t)\right]^{\frac{1}{\alpha}}} .
$$

If $\alpha<\beta$, then, from (37) and (38), for $t \geqslant T_{2}$,

$$
\begin{gathered}
w_{1}^{\prime}(t) \geqslant Q_{1}(t)+w_{1}^{\frac{\beta+1}{\beta}}(t) \frac{\beta\left[-z^{\prime}(t)\right]^{\frac{\beta-\alpha}{\beta}}}{\left[\mathrm{e}^{\int_{t_{0}}^{t} \frac{c(s)}{r(s)} \mathrm{d} s} r(t)\right]^{\frac{1}{\beta}}} \\
=Q_{1}(t)+w_{1}^{\frac{\beta+1}{\beta}}(t) \frac{\beta\left[\mathrm{e}^{\int_{t_{0}}^{t} \frac{c(s)}{r(s)} \mathrm{d} s} r(t)\left(-z^{\prime}(t)\right)^{\alpha}\right]^{\frac{\beta-\alpha}{\alpha \beta}}}{\left[\mathrm{e}^{\int_{t_{0}}^{t} \frac{c(s)}{r(s)} \mathrm{d} s} r(t)\right]^{\frac{1}{\alpha}}} .
\end{gathered}
$$

From (34) for $t \geqslant T_{2},\left[\mathrm{e}^{\int_{t_{0}}^{t} \frac{c(s)}{r(s)} \mathrm{d} s} r(t)\left(-z^{\prime}(t)\right)^{\alpha}\right]^{\prime}>0$, therefore

$$
\begin{aligned}
& {\left[\mathrm{e}^{\int_{t_{0}}^{t} \frac{c(s)}{r(s)} \mathrm{d} s} r(t)\left(-z^{\prime}(t)\right)^{\alpha}\right]^{\frac{\beta-\alpha}{\alpha \beta}}} \\
& \quad \geqslant\left[\mathrm{e}^{\int_{t_{0}}^{T_{2}} \frac{c(s)}{r(s)} \mathrm{d} s} r\left(T_{2}\right)\left(-z^{\prime}\left(T_{2}\right)\right)^{\alpha}\right]^{\frac{\beta-\alpha}{\alpha \beta}}=m_{4} .
\end{aligned}
$$


Clearly, $m_{4}>0$. From (42) and (43), for $t \geqslant T_{2}$,

$$
w_{1}^{\prime}(t) \geqslant Q_{1}(t)+w_{1}^{\frac{\beta+1}{\beta}}(t) \frac{\beta m_{4}}{\left[\mathrm{e}^{\int_{t_{0}}^{t} \frac{c(s)}{r(s)} \mathrm{d} s} r(t)\right]^{\frac{1}{\alpha}}} .
$$

It follows from (41) and (44) that there is $M \in(0,1]$ such that for $t \geqslant T_{2}$,

$$
w_{1}^{\prime}(t) \geqslant Q_{1}(t)+w_{1}^{\frac{\mu+1}{\mu}}(t) \frac{\beta M}{\left[\mathrm{e}^{\int_{t_{0}}^{t} \frac{c(s)}{r(s)} \mathrm{d} s} r(t)\right]^{\frac{1}{\alpha}}} .
$$

Further, it follows from $\left(\mathrm{H}_{1}\right)$ that for $t \geqslant T_{2}$,

$$
\begin{aligned}
\Pi^{\mu}(t) w_{1}^{\prime}(t) & \geqslant \Pi^{\mu}(t) Q_{1}(t) \\
& +\Pi^{\mu}(t) w_{1}^{\frac{\mu+1}{\mu}}(t) \frac{\beta M}{\left[\mathrm{e}^{\int_{t_{0}}^{t} \frac{c(s)}{r(s)} \mathrm{d} s} r(t)\right]^{\frac{1}{\alpha}}} .
\end{aligned}
$$

Integrating (46) from $T_{2}$ to $t$, we get

$$
\begin{aligned}
& \int_{T_{2}}^{t} \Pi^{\mu}(s) w_{1}^{\prime}(s) \mathrm{d} s \geqslant \int_{T_{2}}^{t} \Pi^{\mu}(s) Q_{1}(s) \mathrm{d} s \\
& +\int_{T_{2}}^{t}\left[\Pi^{\mu}(s) w_{1}^{\frac{\mu+1}{\mu}}(s) \frac{\beta M}{\left[\mathrm{e}^{\int_{t_{0}}^{s} \frac{c(k)}{r(k)} \mathrm{d} k} r(s)\right]^{\frac{1}{\alpha}}}\right] \mathrm{d} s .
\end{aligned}
$$

Then

$$
\begin{aligned}
& \Pi^{\mu}(t) w_{1}(t)-\Pi^{\mu}\left(T_{2}\right) w_{1}\left(T_{2}\right) \\
& \geqslant \int_{T_{2}}^{t}\left[\Pi^{\mu}(s)\right]^{\prime} w_{1}(s) \mathrm{d} s+\int_{T_{2}}^{t} \Pi^{\mu}(s) Q_{1}(s) \mathrm{d} s \\
& \quad+\int_{T_{2}}^{t}\left[\Pi^{\mu}(s) w_{1}^{\frac{\mu+1}{\mu}}(s) \frac{\beta M}{\left[\mathrm{e}^{\int_{t_{0}}^{s} \frac{c(k)}{r(k)} \mathrm{d} k} r(s)\right]^{\frac{1}{\alpha}}}\right] \mathrm{d} s .
\end{aligned}
$$

From Lemmas 1 and 2, for $t \geqslant \max \left\{T, T_{2}\right\}$, we have

$$
\begin{aligned}
L- & \Pi^{\mu}\left(T_{2}\right) w_{1}\left(T_{2}\right) \geqslant \int_{T_{2}}^{t} \Pi^{\mu}(s) Q_{1}(s) \mathrm{d} s \\
& -\int_{T_{2}}^{t} \mu \Pi^{\mu-1}(s)\left[\mathrm{e}^{\int_{t_{0}}^{s} \frac{c(k)}{r(k)} \mathrm{d} k} r(s)\right]^{-\frac{1}{\alpha}} w_{1}(s) \mathrm{d} s \\
& -\int_{T_{2}}^{t} \Pi^{\mu}(s) \beta M\left[\mathrm{e}^{\int_{t_{0}}^{s} \frac{c(k)}{r(k)} \mathrm{d} k} r(s)\right]^{-\frac{1}{\alpha}} w_{1}^{\frac{\mu+1}{\mu}}(s) \mathrm{d} s \\
= & \int_{T_{2}}^{t} \Pi^{\mu}(s) Q_{1}(s) \mathrm{d} s-\int_{T_{2}}^{t} \Pi^{\mu-1}(s)\left[\mathrm{e}^{\int_{t_{0}}^{s} \frac{c(k)}{r(k)} \mathrm{d} k} r(s)\right]^{-\frac{1}{\alpha}} \\
\geqslant & \int_{T_{2}}^{t} \Pi^{\mu}(s) Q_{1}(s) \mathrm{d} s \\
& \left.-\int_{T_{2}}^{t} \frac{\mu^{2 \mu+1}}{(\beta M)^{\mu} \Pi(s)(\mu+1)^{\mu+1}\left[\mathrm{e}^{\int_{t_{0}}^{s} \frac{c(k)}{r}(k)} \mathrm{d} k\right.} r(s)\right]^{\frac{1}{\alpha}} \mathrm{d} s .
\end{aligned}
$$

Letting $t \rightarrow \infty$ in (48), it follows from condition (ii) that $L \geqslant \infty$. This is a contradiction. Hence, (7) is oscillatory. This completes the proof.

Remark 1 If $q(t, \xi)=\frac{1}{b-a} q(t), g(t, \xi)=\sigma(t)$, $c(t) \equiv 0$, then (6) reduces to (2). Further, Theorem 1 reduces to Theorem 1 in Ref. 22. In addition, Theorem 2.1 in Refs. 20, 21 requires the condition $\alpha \geqslant \beta$; Theorem 3.1 in Ref. 21 requires the condition $\beta \geqslant \alpha$. Hence, Theorem 1 improves and generalizes these results.

Remark 2 If $q(t, \xi)=\frac{1}{b-a} q(t), g(t, \xi)=\sigma(t)$, then (6) reduces to (4). Theorems 1 and 4 in Ref. 24 require the condition $\alpha \geqslant \beta$ and $\beta \geqslant \alpha$, respectively. Hence, Theorem 1 improves these theorems.

\section{EXAMPLES}

Example 1 Consider the following equation

$$
\begin{aligned}
& {\left[t^{\frac{\alpha}{2}}\left|z^{\prime}(t)\right|^{\alpha-1} z^{\prime}(t)\right]^{\prime}+\frac{\alpha}{2} t^{\frac{\alpha}{2}-1}\left|z^{\prime}(t)\right|^{\alpha-1} z^{\prime}(t)} \\
& +\int_{\frac{1}{2}}^{1} t^{\frac{\alpha}{2}} \xi|x(t \xi)|^{\beta-1} x(t \xi) \mathrm{d} \xi=0,
\end{aligned}
$$

where $t \in[1, \infty), z(t)=x(t)+x(t-1) / 2, \alpha, \beta>0$, $r(t)=t^{\frac{\alpha}{2}}, c(t)=\frac{\alpha}{2} t^{\frac{\alpha}{2}-1}, p(t)=1 / 2, q(t, \xi)=t^{\frac{\alpha}{2}} \xi$, $g(t, \xi)=t \xi$, and $\tau(t)=t-1$.

It is easy to see that

$$
\begin{gathered}
\lim _{t \rightarrow \infty} g(t, \xi)=\infty, 0 \leqslant g(t, \xi) \leqslant t, g_{t}(t, \xi)=\xi>0, \\
g_{\xi}(t, \xi)=t>0, \quad Q(t)=3\left(\frac{1}{2}\right)^{\beta+3} t^{\alpha} .
\end{gathered}
$$


Further,

$$
\begin{aligned}
\lim _{t \rightarrow \infty} R(t) & =\lim _{t \rightarrow \infty} \int_{1}^{t}\left[r(s) \mathrm{e}^{\int_{t_{0}}^{s} \frac{c(k)}{r(k)} \mathrm{d} k}\right]^{-\frac{1}{\alpha}} \mathrm{d} s \\
& =\lim _{t \rightarrow \infty} \int_{1}^{t} \frac{1}{s} \mathrm{~d} s=\infty .
\end{aligned}
$$

So $\left(\mathrm{H}_{3}\right)$ holds. Choose $\rho(t)=t$. For any $m \in(0,1]$, we have

$$
\begin{aligned}
& \int_{1}^{\infty}\left[\rho(t) Q(t)-\frac{r(t)\left[\rho^{\prime}(t)\right]^{\lambda+1} \mathrm{e}^{\int_{t_{0}}^{t} \frac{c(s)}{r(s)} \mathrm{d} s}}{(\lambda+1)^{\lambda+1}\left(m g_{t}(t, a) \rho(t)\right)^{\lambda}}\right] \mathrm{d} t \\
& =\int_{1}^{\infty}\left[3\left(\frac{1}{2}\right)^{\beta+3} t^{\alpha+1}-\frac{2^{\lambda}}{m^{\lambda}(\lambda+1)^{\lambda+1}} t^{\alpha-\lambda}\right] \mathrm{d} t \\
& =\left[\left(\frac{1}{2}\right)^{\beta+3} \frac{3 t^{\alpha+2}}{\alpha+2}-\frac{2^{\lambda} t^{\alpha-\lambda+1}}{m^{\lambda}(\alpha-\lambda+1)(\lambda+1)^{\lambda+1}}\right]_{1}^{\infty}=\infty .
\end{aligned}
$$

Therefore, $\left(\mathrm{H}_{4}\right)$ holds. Hence, the conditions of Theorem 1 are all satisfied. Thus, (49) is oscillatory.

Example 2 Consider the following equation

$$
\begin{aligned}
& {\left[t^{\alpha}\left|z^{\prime}(t)\right|^{\alpha-1} z^{\prime}(t)\right]^{\prime}+\alpha t^{\alpha-1}\left|z^{\prime}(t)\right|^{\alpha-1} z^{\prime}(t)} \\
& +\int_{\frac{1}{2}}^{1} t^{\mu} \xi|x(t \xi)|^{\beta-1} x(t \xi) \mathrm{d} \xi=0,
\end{aligned}
$$

where $t \in[1, \infty), z(t)=x(t)+x(t-1) / 2, \alpha, \beta>0$, $\mu=\max \{\alpha, \beta\}, r(t)=t^{\alpha}, p(t)=1 / 2, c(t)=\alpha t^{\alpha-1}$, $q(t, \xi)=t^{\mu} \xi, g(t, \xi)=t \xi$, and $\tau(t)=t-1$.

It is easy to see that

$$
\begin{gathered}
\lim _{t \rightarrow \infty} g(t, \xi)=\infty, 0 \leqslant g(t, \xi) \leqslant t, g_{t}(t, \xi)=\xi>0, \\
g_{\xi}(t, \xi)=t>0, \quad Q(t)=3\left(\frac{1}{2}\right)^{\beta+3} t^{\alpha+\mu} .
\end{gathered}
$$

Further,

$$
\begin{aligned}
\Pi(t)= & \int_{t}^{\infty}\left[r(s) \mathrm{e}^{\int_{t_{0}}^{s} \frac{c(k)}{r(k)} \mathrm{d} k}\right]^{-\frac{1}{\alpha}} \mathrm{d} s \\
& =\int_{t}^{\infty}\left[s^{\alpha} s^{\alpha}\right]^{-\frac{1}{\alpha}} \mathrm{d} s=\int_{t}^{\infty} s^{-2} \mathrm{~d} s=\frac{1}{t}<\infty .
\end{aligned}
$$

So $\left(\mathrm{H}_{5}\right)$ holds. Choose $\rho(t)=t$. For any $m \in(0,1]$, we have

$$
\begin{aligned}
& \int_{1}^{\infty}\left[\rho(t) Q(t)-\frac{r(t)\left[\rho^{\prime}(t)\right]^{\lambda+1} \mathrm{e}^{\int_{t_{0}}^{t} \frac{c(s)}{r(s)} \mathrm{d} s}}{(\lambda+1)^{\lambda+1}\left(m \rho(t) g_{t}(t, a)\right)^{\lambda}}\right] \mathrm{d} t \\
& =\int_{1}^{\infty}\left[3\left(\frac{1}{2}\right)^{\beta+3} t^{\alpha+\mu+1}-\frac{2^{\lambda}}{m^{\lambda}(\lambda+1)^{\lambda+1}} t^{2 \alpha-\lambda}\right] \mathrm{d} t \\
& \geqslant \int_{1}^{\infty}\left[3\left(\frac{1}{2}\right)^{\beta+1} t^{2 \alpha+1}-\frac{2^{\lambda}}{m^{\lambda}(\lambda+1)^{\lambda+1}} t^{2 \alpha-\lambda}\right] \mathrm{d} t \\
& =\left[\left(\frac{1}{2}\right)^{\beta+1} \frac{3 t^{2 \alpha+2}}{2 \alpha+2}-\frac{2^{\lambda} t^{2 \alpha-\lambda+1}}{(2 \alpha-\lambda+1) m^{\lambda}(\lambda+1)^{\lambda+1}}\right]_{1}^{\infty}=\infty .
\end{aligned}
$$

So $\left(\mathrm{H}_{4}\right)$ holds. In addition, for any $M \in(0,1]$,

$$
\begin{aligned}
& \int_{1}^{\infty} \Pi^{\mu}(t) Q_{1}(t) \mathrm{d} t \\
& \quad-\int_{1}^{\infty} \frac{\mu^{2 \mu+1}}{(\mu+1)^{\mu+1}(\beta M)^{\mu} \Pi(t)\left[r(t) \mathrm{e}^{\int_{t_{0}}^{t} \frac{((s)}{r(s)} \mathrm{d} s}\right]^{\frac{1}{\alpha}}} \mathrm{d} t \\
& =\int_{1}^{\infty}\left[3\left(\frac{1}{2}\right)^{\beta+1} t^{\alpha}-\frac{\mu^{2 \mu+1}}{(\mu+1)^{\mu+1}(M \beta)^{\mu}} t^{-1}\right] \mathrm{d} t \\
& =\left[3\left(\frac{1}{2}\right)^{\beta+1} \frac{1}{\alpha+1} t^{\alpha+1}-\frac{\mu^{2 \mu+1}}{(\mu+1)^{\mu+1}(M \beta)^{\mu}} \ln t\right]_{1}^{\infty}=\infty .
\end{aligned}
$$

Therefore the conditions of Theorem 2 hold. Hence, (50) is oscillatory.

Acknowledgements: This work was supported by the National Natural Science Foundation of China (No. 11471197).

\section{REFERENCES}

1. Wong JSW (1975) On the generalized Emden-Fowler equation. SIAM Rev 17, 339-360.

2. Berezovskaya FS (1997) Properties of the EmdenFowler equation under stochastic disturbances that depend on parameters. $J$ Math Sci 83, 477-484.

3. Agarwal RP, Bohner M, Li T, Zhang C (2014) Oscillation of second-order Emden-Fowler neutral delay differential equations. Ann Mat Pur Appl 193, 1861-1875.

4. Li T, Rogovchenko YV (2017) Oscillation criteria for second-order superlinear Emden-Fowler neutral differential equations. Monatsh Math 184, 489-500.

5. Rogovchenko YV, Tuncay F (2008) Oscillation criteria for second-order nonlinear differential equations with damping. Nonlinear Anal TMA 69, 208-221.

6. Baculíková B, Džurina J (2012) Oscillation theorems for higher order neutral differential equations. Appl Math Comput 219, 3769-3778.

7. Klimas C, Stowe D (2015) Oscillation and integral norms of coefficients in second-order differential equations. J Math Anal Appl 425, 451-459. 
8. Zhang C, Li T, Sun B, Thandapani E (2011) On the oscillation of higher-order half-linear delay differential equations. Appl Math Lett 24, 1618-1621.

9. Baculíková B, Džurina J (2017) Oscillation of functional trinomial differential equations with positive and negative term. Appl Math Comput 295, 47-52.

10. Li T, Rogovchenko YV (2017) On asymptotic behavior of solutions to higher-order sublinear EmdenFowler delay differential equations. Appl Math Lett 67, 53-59.

11. Li T, Rogovchenko YV (2015) Oscillation of secondorder neutral differential equations. Math Nachr 288, 1150-1162.

12. Agarwal RP, Zhang C, Li T (2016) Some remarks on oscillation of second-order neutral differential equations. Appl Math Comput 274, 178-181.

13. Li T, Rogovchenko YV (2016) Oscillation criteria for even-order neutral differential equations. Appl Math Lett 61, 35-41.

14. Li H, Han Z, Sun S (2017) The distribution of zeros of oscillatory solutions for second order nonlinear neutral delay differential equations. Appl Math Lett 63, 14-20.

15. Zhang C, Li T (2013) Some oscillation results for second-order nonlinear delay dynamic equations. Appl Math Lett 26, 1114-1119.

16. Li T, Saker SH (2014) A note on oscillation criteria for second-order neutral dynamic equations on isolated time scales. Commun Nonlinear Sci Numer Simul 19, 4185-4188.
17. Agarwal RP, Bohner M, Li T (2015) Oscillatory behavior of second-order half-linear damped dynamic equations. Appl Math Comput 254, 408-418.

18. Bohner M, Li T (2015) Kamenev-type criteria for nonlinear damped dynamic equations. Sci China Math 58, 1445-1452.

19. Baculíková B, Džurina J (2011) Oscillation theorems for second-order nonlinear neutral differential equations. Comput Math Appl 62, 4472-4478.

20. Liu H, Meng F, Liu P (2012) Oscillation and asymptotic analysis on a new generalized Emden-Fowler equation. Appl Math Comput 219, 2739-2748.

21. Zeng Y, Lou L, Yu Y (2015) Oscillation for EmdenFowler delay differential equations of neutral type. Acta Math Sci 35A, 803-814.

22. Wu Y, Yu Y, Zhang J, Xiao J (2016) Oscillation criteria for second order Emden-Fowler functional differential equations of neutral type. J Inequal Appl 2016, ID 328, 1-11.

23. Bohner M, Saker SH (2006) Oscillation of damped second order nonlinear delay differential equations of Emden-Fowler type. Adv Dyn Syst Appl 1, 163-182.

24. Zeng Y, Li Y, Luo L, Luo Z (2016) Oscillation of generalized neutral delay differential equations of Emden-Fowler type with damping. $J$ Zhejiang Univ (Sci A) 43, 394-400. [in Chinese]

25. Qin HZ, Lu Y (2008) Oscillation criteria for second order differential equations with distributed deviating arguments and a damping term. Int J Pure Appl Math 48, 103-116. 